\title{
Mechanisms of self-screening in pure ice
}

\author{
I. Chikina ${ }^{1}$ and V. Shikin ${ }^{2}$ \\ ${ }^{1}$ CEA Saclay, CEA CNRS, LIONS DSM IRAMIS NIMBE, UMR 3299, F-91191 Gif Sur Yvette, France \\ ${ }^{2}$ Institute of Solid State Physics of RAS, 142432, Chernogolovka, Russia \\ E-mail: shikinv@yandex.ru
}

Received November 24, 2014, published online April 23, 2015

\begin{abstract}
Possible approaches to a correct account of the Coulomb interaction in system of proton-hydroxyl pairs in pure ice (a proton version of intrinsic semiconductor) are discussed for a possibility to evaluate correlation properties of semiconducting media.
\end{abstract}

PACS: 61.20.Qg Structure of associated liquids: electrolytes, molten salts, etc.

Keywords: charges, screening, dissociation.

From the viewpoint of its conduction properties, pure ice possesses all properties of a classical wide gap semiconductor [1]. Its conductivity demonstrates a clear activation-type behavior with the activation energy $\Delta \gg>T$ ( $\Delta$ coincides with the intrinsic gap in the energy spectrum of pure ice, $T$ is the temperature measured in energy units).

Proton-hydroxyl pairs, which exist in pure ice due to the activation decay of $\mathrm{H}_{2} \mathrm{O}$ molecules, interact among themselves in a nontrivial way, necessitating an accurate account of screening effects. Charged systems of low densities $n_{\text {ice }}^{ \pm}<<b^{-3}$ ( $b$ is the lattice spacing), in which carrier are characterized by a relatively large (compared to electrons) effective masses, support a typical self-screening of the long-range Coulomb interaction. The problems involving evaluation of the dissociation degree, mobility, and collective phenomena (Wigner crystallization) need either a proper accounting of the self-screening or a justification for its neglect. In this paper we discuss possible mechanisms for classical self-screening.

The self-screening effect is well known (e.g., see Ref. 2). The relevant energy is

$$
E_{\text {coul }}=\frac{1}{2} V \sum_{a} e z_{a} n_{a} \varphi_{a}, \quad \sum_{a} z_{a} n_{a}=0 .
$$

Here $z_{a}$ is the charge of ions of type $a, n_{a}$ is their concentration, $\varphi_{a}$ is the potential of the field acting upon the ion of type $a$ due to the presence of all other charges.

Expression (1) contains divergent contributions of charged ions of different signs, each of them compensating to a large extent the divergency arising due to the oppositely charged ions. For brevity, this mutual compensation effect is referred to as "self-screening".
The existence of energy (1) is important for various aspects of the theory of many-particle systems with Coulomb interaction. The relevant areas involve properties of ion lattices [3], correlation phenomena in classical plasma, semiconductors, and electrolytes [2], Coulomb (Wigner) crystallization [4], etc. Not all applications of the general formalism have been developed in sufficient detail. The results in this paper are relevant to the dissociation problem and correlation phenomena in pure ice. Here the available literature [1] completely ignores the energy given by Eq. (1).

1. One of the scenarios of self-screening is as follows (the Debye-Hückel scheme, see Ref. 2). We place one of the ions at the origin and allow the other ions to screen it:

$$
\begin{gathered}
\Delta \varphi=\frac{4 \pi}{\varepsilon} \sigma(r), \quad \sigma(r)=|e|\left[n_{+}(r)-n_{-}(r)\right], \\
n_{ \pm}(r)=n_{0} \exp \left[e_{ \pm} \varphi(r) / T\right] .
\end{gathered}
$$

By expanding Eq. (2) in the small parameter $e \varphi(r)<T$, the set (2), (3) transforms into an equation linear in $\varphi(r)$ :

$$
\Delta \varphi=\varphi / \lambda_{D}^{2}, \quad \lambda_{D}^{-2}=\kappa_{D}^{2}, \quad \kappa_{D}^{2}=\frac{8 \pi e^{2}}{\varepsilon T} n_{0} .
$$

The solution to Eq. (4), which satisfies the boundary conditions

$$
r \varphi(r)_{\mid r \rightarrow 0} \rightarrow Z, \quad \varphi(r)_{\mid r \rightarrow \infty} \rightarrow 0,
$$

is

$$
\varphi(r)=e \exp \left(-\kappa_{D} r\right) / r, \quad \kappa_{D}^{2}=\frac{8 \pi e^{2}}{\varepsilon T} n_{0} .
$$

In the expansion of $\varphi(r)$ (6) for small $r$

$$
e \varphi(r) \simeq \frac{e^{2}}{r}-e^{2} \kappa_{D}+\ldots
$$


the first term is the self-energy of the central ion. The second term represents [2] the required correlation correction to the energy of the interaction between the charges in the ionized state:

$$
U_{\text {corr }} \sim e^{2} \kappa_{D} .
$$

Equations (2)-(8) form the basis for all correlation phenomena in classical systems with Coulomb interaction. It is easy to show that Eq. (8) also provides a real value of the energy (1) per pair of ions.

2. It is worthwhile to consider an alternative estimate for $U_{\text {corr }}$ borrowed from the theory of ion lattices [3]. If an ensemble of dissociation-related charges is arranged in a regular lattice suitable to be treated using the Ewald rules, then the energy $U_{\text {corr }}$ per ion pair can be represented as

$$
U_{\text {corr }} \sim \gamma e^{2} / R_{\text {ice }}, \quad R_{\text {ice }} \sim\left(n_{0}\right)^{-1 / 3},
$$

where $\gamma$ is a Madelung constant which depends on the lattice type and $n_{0}$ is the ionized fraction density from (3).

The expressions (8) and (9) for the correlation energy $U_{\text {corr }}$ have different structures and are differently substantiated. Equation (8) is commonly accepted and regularly manifests itself in numerous correlation effects typical of cold plasma and electrolytes (e.g., Coulomb corrections to osmotic pressure [2]). However, derivation of Eq. (8) involves a violation of the translational symmetry in the generally homogeneous system. In addition, the simplifications made to obtain Eq. (4) from Eqs. (2) and (3) also do not add convincing arguments.
Equation (9) actually also relies on model considerations (arrangement of ions into a lattice is equivalent to substitution of a disordered and on the whole neutral system of ions by a fictitious crystal structure). However, this procedure does not violate the homogeneity on the whole. In addition, derivation of Eq. (9) does not require the inequality $e \varphi(r)<T$, which is certainly violated in real systems. Most likely, the difference between Eqs. (8) and (9) can be discovered in a properly designed experiment.

3. Summary. The present report considers the estimate for the correlation energy $U_{\text {corr }}$ in a classical system of interacting ions. In addition to expression (8) for $U_{\text {corr }}$, available in the literature, an alternative estimate (9) is suggested, based on rather general arguments. The difference between (8) and (9) can be detected by accurate measurements of the dissociation degree of donors in pure ice or other intrinsic semiconductors. To the best of our knowledge, no such data are currently available.

This work was partly supported by the RFBR Grant N 15-02-04706 and the Program of the Presidium of RAS.

1. N. Fletcher, The Chemical Physics of Ice, Cambridge, University Press (1970).

2. L. Landau and E. Lifshits, Statistical Physics, Nauka, Moscow (1995) (in Russian).

3. M. Born and Kun Huang, Dynamical Theory of Crystal Lattices, Oxford, Clarendon Press (1954).

4. P.M. Chaikin and T.C. Lubensky, Principles of Condensed Matter Physics, Cambridge, University Press (1997). 